\title{
Quantum transport signatures of chiral edge states in $\mathrm{Sr}_{2} \mathrm{RuO}_{4}$
}

\author{
Rakesh P. Tiwari, ${ }^{1}$ W. Belzig, ${ }^{2}$ Manfred Sigrist, ${ }^{3}$ and C. Bruder ${ }^{1}$ \\ ${ }^{1}$ Department of Physics, University of Basel, Klingelbergstrasse 82, CH-4056 Basel, Switzerland \\ ${ }^{2}$ Department of Physics, University of Konstanz, D-78457 Konstanz, Germany \\ ${ }^{3}$ Theoretische Physik, ETH Zürich, CH-8093 Zürich, Switzerland
}

(Received 21 August 2013; published 28 May 2014)

\begin{abstract}
We investigate transport properties of a double quantum dot based a Cooper pair splitter, where the superconducting lead consists of $\mathrm{Sr}_{2} \mathrm{RuO}_{4}$. The proposed device can be used to explore the symmetry of the superconducting order parameter in $\mathrm{Sr}_{2} \mathrm{RuO}_{4}$ by testing the presence of gapless chiral edge states, which are predicted to exist if the bulk superconductor is described by a chiral $p$-wave state. The odd orbital symmetry of the bulk order parameter ensures that we can realize a regime where the electrons tunneling into the double-dot system come from the chiral edge states and thereby leave their signature in the conductance. The proposed Cooper pair splitter has the potential to probe the chirality of the order parameters in topological superconductors.
\end{abstract}

DOI: 10.1103/PhysRevB.89.184512 PACS number(s): 74.70.Pq, 73.20.-r, 74.45.+c, 85.35.Be

\section{INTRODUCTION}

Unconventional Cooper pairing features a rich phenomenology in superconductivity ranging from nonstandard pairing mechanism to topologically nontrivial phases. An important and much studied example is $\mathrm{Sr}_{2} \mathrm{RuO}_{4}$ which realizes most likely a chiral $p$-wave phase, the quasi-twodimensional analog of the $A$ phase of superfluid ${ }^{3} \mathrm{He}$ [1-3]. This phase has topological character giving rise to chiral edge states (see, for example, Refs. [4,5]). Experimental evidence for chiral $p$-wave pairing can be found in muon spin-relaxation measurements showing broken time-reversal symmetry [6], Knight shift data demonstrating in-plane equal-spin pairing [7], and several more experiments [2]. While edge states have been detected as zero-bias anomalies in tunneling experiments [8-10], the search for the magnetic fields due to the currents induced by the chiral edge states has turned out only negative results so far $[11,12]$. This discrepancy has recently led to a renewed debate on the pairing symmetry realized in $\mathrm{Sr}_{2} \mathrm{RuO}_{4}$ [13].

In the present study we adopt the chiral $p$-wave symmetry for the superconducting phase of $\mathrm{Sr}_{2} \mathrm{RuO}_{4}$ to study consequences in a special quantum transport device. The chiral $p$-wave phase is characterized through a pair wave function having a nonvanishing angular momentum along the $z$ axis, $L_{z}= \pm 1$, and a spin-triplet configuration with $S_{z}=0$ (in-plane equal-spin pairing). In the standard $\mathbf{d}$-vector notation [3], this order parameter can be written as $\hat{\Delta}(\mathbf{k})=i \mathbf{d}(\mathbf{k}) \cdot \boldsymbol{\sigma} \sigma^{y}$, where $\sigma^{i}$ represent the Pauli matrices,

$$
\mathbf{d}(\mathbf{k})=\hat{z} \Delta \frac{k_{x} \pm i k_{y}}{k_{F}},
$$

and $k_{F}$ is the Fermi wave vector. Note that we use the coordinate frame of the tetragonal crystal of $\mathrm{Sr}_{2} \mathrm{RuO}_{4}$ with the $z$ axis parallel to the fourfold crystalline symmetry axis. Although $\mathrm{Sr}_{2} \mathrm{RuO}_{4}$ is a three-band metal there are strong indications that only one band, the genuinely two-dimensional $\gamma$ band, dominates the superconductivity $[14,15]$ so that it is justified to use a single-band picture in the following.

The topological index theorem necessitates the presence of gapless chiral edge modes at the interface of such a chiral superconductor and vacuum. In this article we investigate the possibility of using quantum transport measurements to directly probe these edge states. A schematic view of the proposed device consisting of a double quantum dot based Cooper pair splitter (CPS) is shown in Fig. 1. The double-dot (DD) system we consider is based on two single-walled carbon nanotubes (or one bent nanotube), and the superconducting electrode consists of a thin platelet of $\mathrm{Sr}_{2} \mathrm{RuO}_{4}$. A scanning electron microscope image of an actual device employing a singlet superconductor is shown in Fig. 1 of Ref. [16]. Using a tunnel Hamiltonian approach we calculate the coupling rates from the edge modes and the bulk superconductor to the DD. We show that the subgap quantum transport properties of such a DD tunnel coupled to a $\mathrm{Sr}_{2} \mathrm{RuO}_{4}$ electrode and two normal leads reveal direct information about the presence of chiral edge states in $\mathrm{Sr}_{2} \mathrm{RuO}_{4}$ in the presence of a weak magnetic field.

\section{MODEL HAMILTONIAN}

The total Hamiltonian of the system can be written as

$$
\mathcal{H}=\mathcal{H}_{\mathrm{DD}}+\mathcal{H}_{\mathrm{NL}}+\mathcal{H}_{\mathrm{NR}}+\mathcal{H}_{\mathrm{S}}+\mathcal{H}_{\mathrm{SD}}+\mathcal{H}_{\mathrm{ND}},
$$

where

$$
\begin{aligned}
\mathcal{H}_{\mathrm{DD}}= & \sum_{i, \tau, \sigma}\left(\epsilon+\tau \sigma \Delta_{\mathrm{SO}}\right) n_{i \tau \sigma}+\mathcal{H}_{\mathrm{int}} \\
& +\Delta_{K K^{\prime}} \sum_{i, \sigma}\left(d_{i K \sigma}^{\dagger} d_{i K^{\prime} \sigma}+d_{i K^{\prime} \sigma}^{\dagger} d_{i K \sigma}\right)
\end{aligned}
$$

describes the DD Hamiltonian [17] with $i \in\{L, R\}$ denoting the left and the right dot, and $\sigma \in\{\uparrow, \downarrow\}$ (or equivalently $\sigma \in\{+,-\}$ in algebraic expressions) label the electronic spin states. In addition, $\tau \in\left\{K, K^{\prime}\right\}$ (or equivalently $\tau \in\{+,-\}$ in algebraic expressions) label the electronic orbital states, a notation reminiscent of the valley degeneracy of graphene. The term $\mathcal{H}_{\text {int }}$ accounts for the Coulomb charging energy. We restrict ourselves to the regime with a single electron in each dot due to a strong intra-dot Coulomb charging energy. The constant $\Delta_{\text {SO }}$ corresponds to an effective spin-orbit coupling [18] and the term $\Delta_{K K^{\prime}}$ describes coupling between the $K$ and $K^{\prime}$ orbitals of dot $i$ due to disorder at the level of the carbon nanotube atomic structure [18-20]. In general, there exists an interdot tunneling term. As the left and the right 


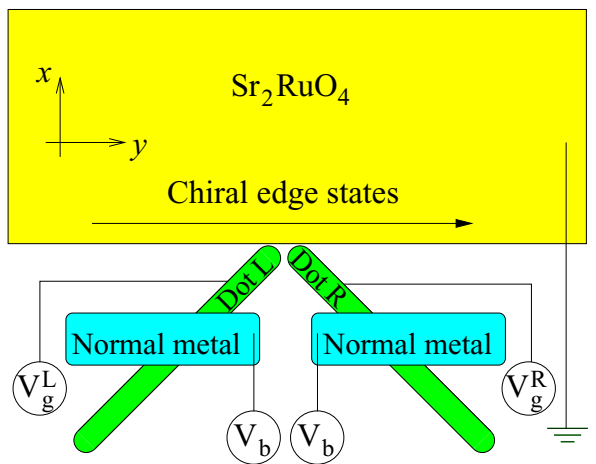

FIG. 1. (Color online) Schematic of the device (not to scale). Two single-walled carbon nanotubes (or one bent nanotube) are used to form a double-dot system tunnel-coupled to a thin platelet of $\mathrm{Sr}_{2} \mathrm{RuO}_{4}$.

dots are assumed to have the same orbital energy $\epsilon$, this term only appears in the double-dot eigenenergies and not in the expressions of the eigenvectors (see Ref. [17]). Thus this term is irrelevant to our discussion and is ignored in the rest of the article. Finally, $d_{i \tau \sigma}^{\dagger}$ denotes the DD creation operator for an electron with spin $\sigma$ in orbital $\tau$ of dot $i$ and $n_{i \tau \sigma}=d_{i \tau \sigma}^{\dagger} d_{i \tau \sigma}$, and $\mathcal{H}_{\mathrm{N} \eta}$ with $\eta \in\{L, R\}$ is the Hamiltonian of the left $(L)$ and the right $(R)$ normal leads

$$
\mathcal{H}_{\mathrm{N} \eta}=\sum_{k \sigma} \epsilon_{\eta k} c_{\eta k \sigma}^{\dagger} c_{\eta k \sigma},
$$

where $k$ represents the orbital state in the normal leads and $c_{\eta k \sigma}^{\dagger}$ denotes the normal lead electron creation operator.

$\mathcal{H}_{\mathrm{S}}$ is the Hamiltonian of the superconducting lead. In our analysis we model the $\mathrm{Sr}_{2} \mathrm{RuO}_{4}$ as a thin platelet with a twodimensional spin-triplet mean-field Hamiltonian with $\mathbf{d} \| \hat{z}$, assuming that the energy band and the order parameter have no momentum dependence in the $z$ direction. Thus

$$
\begin{aligned}
\mathcal{H}_{\mathrm{S}}= & \sum_{\sigma} \psi_{\sigma}^{\dagger}(\boldsymbol{r}) h_{0}(\boldsymbol{r}) \psi_{\sigma}(\boldsymbol{r})+\frac{1}{g}|\boldsymbol{\eta}(\boldsymbol{r})|^{2} \\
& -\frac{i}{2 k_{F}}\left\{\boldsymbol{\eta}(\boldsymbol{r}) \cdot\left[\psi_{\downarrow}^{\dagger}(\boldsymbol{r}) \nabla \psi_{\uparrow}^{\dagger}(\boldsymbol{r})+\psi_{\uparrow}^{\dagger}(\boldsymbol{r}) \nabla \psi_{\downarrow}^{\dagger}(\boldsymbol{r})\right]\right. \\
& \left.+\boldsymbol{\eta}^{*}(\boldsymbol{r}) \cdot\left[\psi_{\downarrow}(\boldsymbol{r}) \nabla \psi_{\uparrow}(\boldsymbol{r})+\psi_{\uparrow}(\boldsymbol{r}) \nabla \psi_{\downarrow}(\boldsymbol{r})\right]\right\},
\end{aligned}
$$

where $h_{0}(\boldsymbol{r})=-\frac{\hbar^{2}}{2 m} \nabla^{2}-\mu_{\mathrm{S}}, \psi_{\sigma}$ is the annihilation operator of an electron with spin $\sigma, g$ is the coupling constant of the attractive interaction that is responsible for $p$-wave pairing $(g>0)$, and $\nabla=\left(\partial_{x}, \partial_{y}\right)$. The superconducting order parameter $\boldsymbol{\eta}$ should satisfy the (self-consistency) gap equation, obtained from $\left(\delta / \delta \eta^{*}\right)\left\langle H_{\mathrm{S}}\right\rangle=0[5]$,

$$
\boldsymbol{\eta}=\left(\eta_{x}, \eta_{y}\right)=\frac{i g}{2 k_{F}}\left\langle\psi_{\uparrow}(\boldsymbol{r})\left[\nabla \psi_{\downarrow}(\boldsymbol{r})\right]-\left[\nabla \psi_{\uparrow}(\boldsymbol{r})\right] \psi_{\downarrow}(\boldsymbol{r})\right\rangle .
$$

For a uniform system with a $\boldsymbol{d}$ vector corresponding to Eq. (1), this implies $\eta=\Delta(1, \pm i)$. Within this model a description of the chiral edge states can be obtained by solving the Bogoliubov-de Gennes (BdG) equation across the boundary of the superconductor, assuming a simplified gap function $\eta=\Theta(x) \Delta(1, \pm i)$, where $\Theta(x)$ is the Heaviside step function [21]. Here, the $x$ direction is assumed to be normal to the boundary of the superconductor. The field operators $\psi_{\sigma}$ can be expanded in terms of Bogoliubov operators as [5]

$$
\left(\begin{array}{l}
\psi_{\uparrow}(\mathbf{r}) \\
\psi_{\downarrow}^{\dagger}(\mathbf{r})
\end{array}\right)=\sum_{\mathbf{k}}\left(\begin{array}{ll}
u_{\mathbf{k}} & v_{\mathbf{k}}^{*} \\
v_{\mathbf{k}} & u_{\mathbf{k}}^{*}
\end{array}\right)\left(\begin{array}{c}
\gamma_{\mathbf{k} \uparrow} \\
\gamma_{\mathbf{k} \downarrow}^{\dagger}
\end{array}\right)
$$

where the Bogoliubov operators are defined as $\gamma_{\mathbf{k} \sigma}|0\rangle=0$, with $|0\rangle$ denoting the ground state of the superconductor. The bound-state solutions of the $\mathrm{BdG}$ equations satisfying the boundary condition $u_{\mathbf{k}}=v_{\mathbf{k}}=0$ at $x=0$ denote the chiral edge states and are given by

$$
\left(\begin{array}{l}
u_{\mathbf{k}}(\mathbf{r}) \\
v_{\mathbf{k}}(\mathbf{r})
\end{array}\right)=\mathcal{N} \exp \left(-\frac{x}{\xi_{0}}+i k_{y} y\right) \sin \left(k_{x} x\right)\left(\begin{array}{c}
e^{i \pi / 4} \\
e^{-i \pi / 4}
\end{array}\right),
$$

with energy eigenvalues $\epsilon_{\chi}\left(k_{y}\right)=\Delta \frac{k_{y}}{k_{F}}$ and the normalization factor $\mathcal{N}=\sqrt{\frac{2}{\xi_{0} L_{y}}}$. Here $L_{y}$ is the length of the superconductor in the $y$ direction. These edge-state solutions decaying in the bulk on the length scale $\xi_{0}=\hbar v_{F} / \Delta$ have been obtained within the Andreev approximation $\left(\Delta \ll \mu_{\mathrm{S}}\right)$.

The tunnel Hamiltonian between dot $l \in\{L, R\}$ and the point $\mathbf{r}_{l}$ in the superconductor is

$$
\mathcal{H}_{\mathrm{SD}}=\sum_{l, \tau} T_{\mathrm{SD}}\left[d_{l \tau \uparrow}^{\dagger} \psi_{\uparrow}\left(\mathbf{r}_{l}\right)+d_{l \bar{\tau} \downarrow}^{\dagger} \psi_{\downarrow}\left(\mathbf{r}_{l}\right)\right]+\text { H.c. },
$$

here $\psi_{\sigma}\left(\mathbf{r}_{l}\right)$ annihilates an electron with spin $\sigma$ at site $\mathbf{r}_{l}$, and $d_{l \tau \sigma}^{\dagger}$ creates it again (with the same spin) at dot $l$ and in electronic orbital $\tau$ with amplitude $T_{\mathrm{SD}}$. Finally,

$$
\mathcal{H}_{\mathrm{ND}}=\sum_{k, \tau, \sigma}\left[T_{\mathrm{NL}} c_{\mathrm{L} k \sigma}^{\dagger} d_{\mathrm{L} \tau \sigma}+T_{\mathrm{NR}} c_{\mathrm{R} k \sigma}^{\dagger} d_{\mathrm{R} \tau \sigma}\right]+\text { H.c. }
$$

describes the tunnel coupling between the left (right) dot and the left (right) normal lead with amplitude $T_{\mathrm{NL}}$ and $T_{\mathrm{NR}}$, respectively.

\section{TUNNELING BETWEEN THE SUPERCONDUCTOR AND THE DD}

In our device the coherent injection of electrons in the DD via tunneling appears in the following order. A Cooper pair breaks up in $\mathrm{Sr}_{2} \mathrm{RuO}_{4}$, one electron with spin $\sigma$ tunnels to one of the dots from the point of the superconductor nearest to this dot. This results in a virtual state where the other electron either creates a quasiparticle with energy $E_{\mathbf{k}}>\Delta$ (referred to as case I below) or it occupies an empty edge state with energy $\epsilon_{\chi}\left(k_{y}\right)$ (referred to as case II below). The second electron with spin $-\sigma$ then tunnels to the other empty dot before the first electron with spin $\sigma$ tunnels out to the normal lead making the tunneling of both electrons almost simultaneous (within the uncertainty time $\hbar / \Delta$ ). Reference [22] describes such a device for singlet superconductors.

To elucidate the role of chiral edge states in the Cooper pair tunneling described above, we derive the effective Hamiltonian of the DD-normal lead system by integrating out the superconductor's degrees of freedom and including terms up to second order in $T_{\mathrm{SD}}$ [22]. Writing $\mathcal{H}=\mathcal{H}_{0}+\mathcal{H}_{\mathrm{SD}}$ we obtain $\mathcal{H}_{\text {eff }}=\mathcal{H}_{\mathrm{DD}}+\mathcal{H}_{\mathrm{NL}}+\mathcal{H}_{\mathrm{NR}}-\mu_{\mathrm{S}}+\mathcal{H}_{\mathrm{SD}}^{\text {eff }}+\mathcal{H}_{\mathrm{ND}}$, where

$$
\mathcal{H}_{\mathrm{SD}}^{\mathrm{eff}}=\lim _{\zeta \rightarrow 0^{+}}\left\langle 0\left|\mathcal{H}_{\mathrm{SD}} \frac{1}{i \zeta-\mathcal{H}_{0}} \mathcal{H}_{\mathrm{SD}}\right| 0\right\rangle \text {. }
$$


We find that

$$
\mathcal{H}_{\mathrm{SD}}^{\mathrm{eff}}=T_{\mathrm{SD}}^{2} \sum_{\tau} t_{e h}\left(d_{\mathrm{R} \tau \uparrow}^{\dagger} d_{\mathrm{L} \bar{\tau} \downarrow}^{\dagger}+d_{\mathrm{R} \bar{\tau} \downarrow}^{\dagger} d_{\mathrm{L} \tau \uparrow}^{\dagger}\right)+\text { H.c. }
$$

where $t_{e h}=t_{e h}^{\mathrm{I}}+t_{e h}^{\mathrm{II}}$ is the effective amplitude for the tunnel coupling between the superconductor and the DD system, including contributions from both the quasiparticle states denoted by $t_{e h}^{\mathrm{I}}$ and the chiral edge states denoted by $t_{e h}^{\mathrm{II}}$. For simplicity we have ignored terms with opposite spin and the same valley index on both dots. The effect of these terms is essentially captured by the $\Delta_{K K^{\prime}}$ term in Eq. (3). Adding these terms to Eq. (12) would only modify our results quantitatively. Within the $\mathrm{BdG}$ theory (and using that for a spin-triplet superconductor $\left.u_{\mathbf{k}} v_{\mathbf{k}}=-u_{-\mathbf{k}} v_{-\mathbf{k}}\right)$ the quasiparticle contribution is

$$
t_{e h}^{\mathrm{I}}=4 i \sum_{\mathbf{k}} \frac{u_{\mathbf{k}} v_{\mathbf{k}}}{E_{\mathbf{k}}} \sin (\mathbf{k} \cdot \delta \mathbf{r}),
$$

where $\delta \mathbf{r}=\mathbf{r}_{\mathrm{R}}-\mathbf{r}_{\mathrm{L}}$ and $E_{\mathbf{k}}^{2}=\Delta^{2}+\xi_{\mathbf{k}}^{2}$ denotes the quasiparticle spectrum. The sum over $\mathbf{k}$ can be performed by linearizing the spectrum around the Fermi level with Fermi wave vector $k_{F}$. Finally we obtain

$$
t_{e h}^{\mathrm{I}}=i 2 \pi \rho_{\mathrm{S}} J_{1}\left(k_{F} \delta r\right),
$$

where $\rho_{\mathrm{S}}$ is the (normal-state) density of states of the superconductor at the Fermi level, $J_{1}\left(k_{F} \delta r\right)$ denotes the first-order Bessel function and $\delta r=\left|\mathbf{r}_{\mathrm{R}}-\mathbf{r}_{\mathrm{L}}\right|$. Note that $\lim _{\delta r \rightarrow 0} t_{e h}^{\mathrm{I}}=0$.

For the chiral edge states the Bogoliubov transformation is given by Eq. (8). A similar calculation yields

$$
t_{e h}^{\mathrm{II}}=2 \lim _{\zeta \rightarrow 0^{+}} \sum_{\mathbf{k}} \frac{u_{\mathbf{k}}\left(\mathbf{r}_{\mathrm{R}}\right) v_{\mathbf{k}}^{*}\left(\mathbf{r}_{\mathrm{L}}\right)}{\epsilon_{\chi}\left(k_{y}\right)+\epsilon-i \zeta},
$$

where $\epsilon$ indicates the double-dot orbital energy, see Eq. (3), and the sum over $\mathbf{k}$ represents a summation over one dimension momentum along the edge. Interestingly we find that this contribution remains finite even when $\mathbf{r}_{\mathrm{L}}=\mathbf{r}_{\mathrm{R}}$. As we are interested in probing the chiral edge states, we consider a CPS device where the two electrons tunneling into the DD system satisfy $\mathbf{r}_{\mathrm{L}} \approx \mathbf{r}_{\mathrm{R}}$ (implying that $t_{e h}^{\mathrm{II}} / t_{e h}^{\mathrm{I}} \rightarrow \infty$ ). Therefore in our device the electron tunneling amplitude between the superconductor and the DD system contains only the edge-state contribution $t_{e h} \approx t_{e h}^{\mathrm{II}}$.

\section{TUNNELING BETWEEN THE NORMAL LEADS AND THE DD}

In the sequential-tunneling regime a convenient description of quantum transport in our device can be obtained within the master-equation formalism. By integrating out the normal-lead degrees of freedom [23], the tunneling of an electron between one quantum dot and the corresponding normal lead is described by a tunnel rate $\Gamma_{\mathrm{N} \eta}=2 \pi \rho_{\eta}\left|T_{\mathrm{N} \eta}\right|^{2}$, where $\eta \in\{L, R\}$ denotes the left $(L)$ and the right $(R)$ lead, and $\rho_{\mathrm{N} \eta}$ denotes the corresponding density of states (assumed to be be constant in the energy window relevant for transport). For simplicity we assume $\Gamma_{\mathrm{NL}}=\Gamma_{\mathrm{NR}}=\Gamma_{\mathrm{N}}$. In the master-equation description of the dynamics of our device, to lowest order in $\Gamma_{\mathrm{N}}$ we only need to consider the occupation probabilities of the eigenstates of the effective Hamiltonian $\mathcal{H}_{\mathrm{eff}}^{\mathrm{DD}}=\mathcal{H}_{\mathrm{DD}}+\mathcal{H}_{\mathrm{SD}}^{\text {eff }}$.
Due to Coulomb blockade, the DD eigenstates participating in transport are the double-dot empty state $|0,0\rangle$, the singly occupied states $|\tau \sigma, 0\rangle=d_{\mathrm{L} \tau \sigma}^{\dagger}|0,0\rangle$ and $|0, \tau \sigma\rangle=d_{\mathrm{R} \tau \sigma}^{\dagger}|0,0\rangle$, and the nonlocal doubly occupied states $\left|\tau \sigma, \tau^{\prime} \sigma^{\prime}\right\rangle=$ $d_{\mathrm{L} \tau \sigma}^{\dagger} d_{\mathrm{R} \tau^{\prime} \sigma^{\prime}}^{\dagger}|0,0\rangle$. For $t_{e h}=0, \Delta_{\mathrm{SO}}=0$, and $\Delta_{K K^{\prime}}=0$, to create the doubly occupied states of $\mathcal{H}_{\text {eff }}^{\mathrm{DD}}$ will cost an energy $2 \epsilon$. In our device we assume $t_{e h} \ll\left\{\Delta_{\text {SO }}, \Delta_{K K^{\prime}}\right\}$, this allows us to isolate a subset of five lowest-energy eigenstates of $\mathcal{H}_{\mathrm{eff}}^{\mathrm{DD}}$ in the regime $\epsilon \sim \Delta_{r}$, which are separated from all other doubly occupied eigenstates by at least $\sim 2 \Delta_{r}$, where $\Delta_{r}=$ $\sqrt{\Delta_{\text {SO }}^{2}+\Delta_{K K^{\prime}}^{2}}$. A similar calculation for a singlet superconductor is presented in Ref. [17] and its supplemental information. These five states include two triplet states $\left|T_{\uparrow \uparrow}\right\rangle,\left|T_{\downarrow \downarrow}\right\rangle$ as well as the singlet state $\left|S_{\uparrow \downarrow}\right\rangle$ with energies $E=2 \epsilon-2 \Delta_{r}$, and the two hybridized states $\left|V_{n}\right\rangle=\sqrt{1-\left|v_{n}\right|^{2}}|0,0\rangle+v_{n}\left|T_{\uparrow \downarrow}\right\rangle$ with energies $E_{n}=\left[\epsilon-\Delta_{r}-(-1)^{n} \sqrt{2\left|t_{e h}\right|^{2}+\left(\epsilon-\Delta_{r}\right)^{2}}\right]$ for $n \in\{1,2\}$. Here, $v_{1}=t_{e h} / \sqrt{2\left|t_{e h}\right|^{2}+\left(\epsilon-\Delta_{r}\right) E_{2}}$ and $v_{2}=$ $t_{e h} / \sqrt{2\left|t_{e h}\right|^{2}+\left(\epsilon-\Delta_{r}\right) E_{1}}$. In our device the coherent injection of Cooper pairs with symmetry as described by Eq. (1) ensures that the triplet states $\left|T_{\uparrow \uparrow}\right\rangle$ and $\left|T_{\downarrow \downarrow}\right\rangle$, as well as the singlet state $\left|S_{\uparrow \downarrow}\right\rangle$, are not populated.

The occupation probabilities $P_{V_{1}}, P_{V_{2}}$, and $P_{\text {single }}$ of the states $\left|V_{1}\right\rangle,\left|V_{2}\right\rangle$, and the global probability of having a singly occupied DD state, respectively satisfy a master equation $d P / d t=M P$. Here $P=\left[P_{V_{1}}, P_{V_{2}}, P_{\text {single }}\right]^{T}$ and to lowest order in $\Gamma_{\mathrm{N}}$ we find

$$
M=\Gamma_{\mathrm{N}}\left[\begin{array}{ccc}
-2\left|v_{1}\right|^{2} & 0 & 1-\left|v_{1}\right|^{2} \\
0 & -2\left|v_{2}\right|^{2} & 1-\left|v_{2}\right|^{2} \\
2\left|v_{1}\right|^{2} & 2\left|v_{2}\right|^{2} & -1
\end{array}\right] .
$$

Here we have assumed that the applied bias voltage $V$ between the superconductor and the normal leads resulting in the subgap transport is such that the single electrons can tunnel from the DD system to the normal leads but not vice versa. By solving the equation $M P_{\text {stat }}=0$, we can calculate the steady-state value of the occupation probabilities $P_{\text {stat }}$ which allows us to compute the dc current response of our device as $I_{\mathrm{CPS}}=R P_{\text {stat }}$, where $R=e \Gamma_{\mathrm{N}}\left[2\left|v_{1}\right|^{2}, 2\left|v_{2}\right|^{2}, 1\right]$. This current response can be understood in terms of state cycles which produce a flow of electrons towards the normal leads [17]. Due to the injection of Cooper pairs the DD system starts in a state $\left|V_{n}\right\rangle$, where $n \in\{1,2\}$. Now a single-electron tunneling event to either of the leads results in a singly occupied state in the DD system, another such tunneling event can then cause a transition back to $\left|V_{n}\right\rangle$ because $\left|V_{n}\right\rangle$ has a $|0,0\rangle$ component. We find to lowest order in $\Gamma_{\mathrm{N}}$

$$
\begin{aligned}
I_{\mathrm{CPS}} & =e \Gamma_{\mathrm{N}} \frac{2\left|t_{e h}\right|^{2}}{2\left|t_{e h}\right|^{2}+\left(\epsilon-\Delta_{r}\right)^{2}} \\
& =e \Gamma_{\mathrm{N}} \frac{2\left(\left|t_{e h}^{\mathrm{I}}\right|^{2}+\left|t_{e h}^{\mathrm{II}}\right|^{2}\right)}{2\left(\left|t_{e h}^{\mathrm{I}}\right|^{2}+\left|t_{e h}^{\mathrm{II}}\right|^{2}\right)+\left(\epsilon-\Delta_{r}\right)^{2}} .
\end{aligned}
$$

Figure 2 shows $I_{\mathrm{CPS}}$ for various values of $t_{e h}$. In the limit $\delta r \rightarrow 0$, i.e., $t_{e h} \approx t_{e h}^{\mathrm{II}}$, the current given in Eq. (17) is solely due to the chiral edge states. Even if this condition is not satisfied, our device can provide a signature of the presence of the chiral edge states. By measuring the full width at half maximum (FWHM), we can extract information about the 


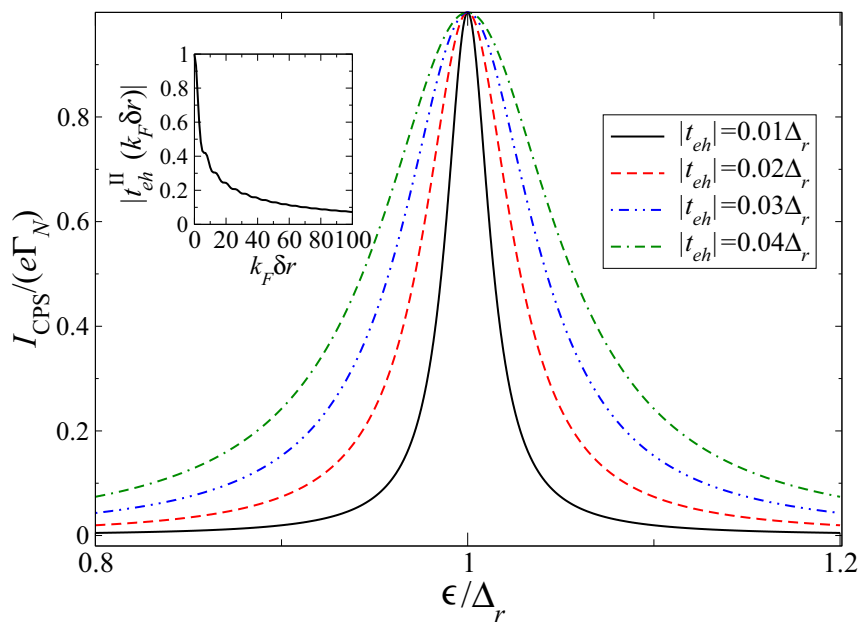

FIG. 2. (Color online) $I_{\mathrm{CPS}}$ as a function of $\epsilon$ that is tunable by (symmetric) external gate voltages, calculated using Eq. (17) for various values of $\left|t_{e h}\right|$. Inset: $\left|t_{e h}^{\mathrm{II}}\left(k_{F} \delta r\right)\right| /\left|t_{e h}^{\mathrm{II}}(0)\right|$ as a function of $k_{F} \delta r$ for $\epsilon / \Delta=0.01$ calculated using Eq. (15).

dependence of $t_{e h}^{\mathrm{I}}$ and $t_{e h}^{\mathrm{II}}$ on various parameters of the device such as $k_{F} \delta r$.

\section{DETECTING CHIRALITY}

Our measuring scheme so far does not allow us to distinguish chiral from nonchiral edge states. We may, however, run a small in-plane supercurrent along the edge of the superconductor (due to Meissner screening it will be concentrated within the London penetration depth). The effect of the current is to increase or decrease the density of states of the edge states depending on the chirality or the direction of the current, respectively [24]. Equation (15) includes a sum over all momenta which can be converted to an integral over energy. The integrand then is directly proportional to the density of states of the chiral edge states. As a consequence $t_{e h}^{\mathrm{II}}$ and thereby the FWHM of the current shown in Fig. 2 would depend essentially linearly on the supercurrent (around their values in its absence), providing a clear signature of chirality. This would make it possible to determine the sign of the chirality of the superconducting phase.

\section{CONCLUSIONS}

We have calculated the current response of a double quantum dot based Cooper pair splitter, where the superconducting electrode is a thin platelet of $\mathrm{Sr}_{2} \mathrm{RuO}_{4}$. The expression for the current response provides direct evidence for the chiral edge states predicted to exist along the boundaries of the superconductor. By applying a small supercurrent along the edge of the superconducting platelet our device can also detect the sign of the chirality. The chiral edge states predicted to exist for $\mathrm{Sr}_{2} \mathrm{RuO}_{4}$ have never been observed. The recent experimental advances in Cooper pair splitter devices (Ref. [16]) can be used to explore the chirality of the bulk superconducting order parameter in $\mathrm{Sr}_{2} \mathrm{RuO}_{4}$. Furthermore, the tunability and sensitivity of double quantum dot devices based on carbon nanotubes makes our proposal attractive for investigating small edge currents. An experimental realization of this device would help settle the debate on the order parameter of superconducting $\mathrm{Sr}_{2} \mathrm{RuO}_{4}$. The chiral edge states discussed here are closely related to the edge states of topological superconductors in general. Thus, the device proposed here could be adapted for detecting chiral edge states of a broad range of topological superconductors.

\section{ACKNOWLEDGMENTS}

We thank A. Cottet for helpful discussions. R.P.T. and C.B. acknowledge financial support by the Swiss SNF and the NCCR Quantum Science and Technology. W.B. acknowledges financial support from the DFG through BE3803/3 and the Baden Württemberg Stiftung.
[1] Y. Maeno, H. Hashimoto, K. Yoshida, S. Nihizaki, T. Fujita, J. G. Bednorz, and F. Lichtenberg, Nature (London) 372, 532 (1994).

[2] A. P. Mackenzie and Y. Maeno, Rev. Mod. Phys. 75, 657 (2003).

[3] A. J. Leggett, Rev. Mod. Phys. 47, 331 (1975).

[4] T. Morinari and M. Sigrist, J. Phys. Soc. Jpn. 69, 2411 (2000).

[5] A. Furusaki, M. Matsumoto, and M. Sigrist, Phys. Rev. B 64, 054514 (2001).

[6] G. M. Luke, Y. Fudamoto, K. M. Kojima, M. I. Larkin, J. Merrin, B. Nachumi, Y. J. Uemura, Y. Maeno, Z. Q. Mao, Y. Mori, H. Nakamura, and M. Sigrist, Nature (London) 394, 558 (1998).

[7] K. Ishida, H. Mukuda, Y. Kitaoka, K. Asayama, Z. Q. Mao, Y. Mori, and Y. Maeno, Nature (London) 396, 658 (1998).

[8] F. Laube, G. Goll, H. von Löhneysen, M. Fogelström, and F. Lichtenberg, Phys. Rev. Lett. 84, 1595 (2000).
[9] Y. Liu, K. Nelson, Z. Mao, R. Jin, and Y. Maeno, J. Low Temp. Phys. 131, 1059 (2003).

[10] S. Kashiwaya, H. Kashiwaya, H. Kambara, T. Furuta, H. Yaguchi, Y. Tanaka, and Y. Maeno, Phys. Rev. Lett. 107, 077003 (2011).

[11] T. Tamegai, K. Yamazaki, M. Tokunaga, Z. Mao, $\begin{array}{llll}\text { and Y. Maeno, Physica C 388-389, } 499 & 4\end{array}$ (2003).

[12] J. R. Kirtley, C. Kallin, C. W. Hicks, E.-A. Kim, Y. Liu, K. A. Moler, Y. Maeno, and K. D. Nelson, Phys. Rev. B 76, 014526 (2007).

[13] C. Kallin, Rep. Prog. Phys. 75, 042501 (2012).

[14] J.-W. Huo, T. M. Rice, and F.-C. Zhang, Phys. Rev. Lett. 110, 167003 (2013).

[15] Q. H. Wang, C. Platt, Y. Yang, C. Honerkamp, F. C. Zhang, W. Hanke, T. M. Rice, and R. Thomale, Europhys. Lett. 104, 17013 (2013). 
[16] L. Hofstetter, S. Csonka, A. Baumgartner, G. Fülöp, S. d'Hollosy, J. Nygård, and C. Schönenberger, Phys. Rev. Lett. 107, 136801 (2011).

[17] A. Cottet, T. Kontos, and A. L. Yeyati, Phys. Rev. Lett. 108, 166803 (2012).

[18] T. S. Jesperson, K. Grove-Rasmussen, J. Paaske, K. Muraki, T. Fujisawa, J. Nygård, and K. Flensberg, Nat. Phys. 7, 348 (2011).

[19] W. Liang, M. Bockrath, and H. Park, Phys. Rev. Lett. 88, 126801 (2002).
[20] F. Kuemmeth, S. Ilani, D. C. Ralph, and P. L. McEuen, Nature 452, 448 (2008).

[21] For a self-consistent description see Ref. [5].

[22] P. Recher, E. V. Sukhorukov, and D. Loss, Phys. Rev. B 63, 165314 (2001).

[23] J. Eldridge, M. G. Pala, M. Governale, and J. König, Phys. Rev. B 82, 184507 (2010).

[24] T. Yokoyama, C. Iniotakis, Y. Tanaka, and M. Sigrist, Phys. Rev. Lett. 100, 177002 (2008). 